\title{
Echocardiographic parameters of left ventricular non-systolic function predict length of stay following coronary artery bypass graft - a prospective observational study
}

samhati Mondal ${ }^{1}$, Nauder Faraday ${ }^{2}$, Weidong $\mathrm{Gao}^{2}$, Sarabdeep Singh ${ }^{2}$, Sachidanand Hebbar $^{2}$, Kimberly Hollander ${ }^{1}$, Thomas Metkus², Lee Goeddel ${ }^{2}$, Maria Bauer ${ }^{2}$, Brian Bush $^{2}$, Brian Cho ${ }^{2}$, Stephanie Cha ${ }^{2}$, Stephanie Ibekwe ${ }^{3}$, Domagoj Mladinov ${ }^{4}$, Noah Rolleri $^{5}$, Laeben Lester ${ }^{2}$, Jochen Steppan ${ }^{2}$, Rosanne Sheinberg ${ }^{2}$, Nadia Hensley ${ }^{2}$, Anubhav Kapoor $^{6}$, and Jeffrey Dodd-o ${ }^{2}$

${ }^{1}$ University of Maryland School of Medicine

${ }^{2}$ Johns Hopkins Medicine

${ }^{3}$ Ben Taub General Hospital

${ }^{4}$ University of Alabama at Birmingham HCOP

${ }^{5}$ University of Pittsburgh Medical Center

${ }^{6}$ Mercy General Hospital

September 27, 2021

\begin{abstract}
Background: Abnormal left ventricular (LV) echocardiographic parameters during non-systolic phase, with or without a diagnosis of heart failure, is a common finding that can be easily diagnosed by intra-operative transesophageal echocardiography (TEE). However, its association with duration of hospital stay after coronary artery bypass (CAB) is unknown. Objective: To determine if Abnormal left ventricular (LV) echocardiographic parameters during non-systolic phase is associated with length of hospital stay after coronary artery bypass surgery (CAB). Method: Prospective observational study at a single tertiary academic medical center Result: Median time to hospital discharge was significantly longer for subjects with abnormal left ventricular (LV) echocardiographic parameters during non-systolic phase (9.1/IQR 6.6-13.5 days) than those with normal LV non-systolic function (6.5/IAR 5.3-9.7days) ( $\mathrm{P}<0.001$ ). The probability of hospital discharge was $34 \%$ lower (HR 0.66/95\% CI 0.47-0.93) for subjects with abnormal LV function even during non-systole despite a normal LV systolic function, independent of potential confounders, including a baseline diagnosis of heart failure Conclusions and Relevance: In patients with normal systolic function undergoing CAB, non-systolic LV dysfunction is associated with prolonged duration of postoperative hospital stay. This association cannot be explained by baseline comorbidities or common post-operative complications.

Echocardiographic parameters of left ventricular nonsystolic function predict length of stay following coronary artery bypass graft - a prospective observational study

Samhati Mondal, $\mathrm{MD}^{1^{*} \#}$; Nauder Faraday, MD, $\mathrm{MPH}^{2 *}$; Wei Dong Gao, MD ${ }^{2}$, Sarabdeep Singh, $\mathrm{PhD}^{3}$; Sachidanand Hebbar, $\mathrm{PhD}^{2}$; Kimberly N. Hollander, $\mathrm{MD}^{1}$; Thomas S. Metkus, MD, $\mathrm{PhD}^{4}$, Lee Goeddel, $\mathrm{MD}, \mathrm{MPH}^{2}$; Maria Bauer, $\mathrm{MD}^{2}$; Brian Bush, $\mathrm{MD}^{2}$; Brian Cho, $\mathrm{MD}^{2}$; Stephanie Cha, $\mathrm{MD}^{2}$; Stephanie O. Ibekwe, $\mathrm{MD}, \mathrm{MPH}^{5}$; Domagoj Mladinov, $\mathrm{MD}, \mathrm{PhD}^{6}$; Noah Rolleri, $\mathrm{MD}^{7}$; Laeben Lester, $\mathrm{MD}^{2}$; Jochen Steppan, $\mathrm{MD}^{2}$; Rosanne Sheinberg, $\mathrm{MD}^{2}$; Nadia Hensley, $\mathrm{MD}^{2}$; Anubhav Kapoor, $\mathrm{MD}^{8}$; Jeffrey M. Dodd-o, $\mathrm{MD}, \mathrm{PhD}^{2}$

${ }^{1}$ Department of Anesthesiology,
\end{abstract}


University of Maryland School of Medicine, Baltimore, MD

${ }^{2}$ Department of Anesthesiology and Critical Care Medicine

Johns Hopkins University, Baltimore, MD

${ }^{3}$ Johns Hopkins Medicine, Baltimore, MD

${ }^{4}$ Division of Cardiology, Department of Medicine, Johns Hopkins University, Baltimore, MD

${ }^{5}$ Department of Anesthesiology, Cardiovascular Division, BTGH

Baylor College of Medicine, Houston, TX

${ }^{6}$ Department of Anesthesiology and Critical Care Medicine

University of Alabama, Birmingham, AL

${ }^{7}$ Department of Anesthesiology and Perioperative Medicine

University of Pittsburgh Medical Center, Pittsburgh, PA

${ }^{8}$ Department of Anesthesiology

Mercy General Hospital, Sacramento, CA

* Co-first authors

\# Corresponding Author

\section{Corresponding Author:}

\section{Samhati Mondal, MBBS, MD}

Assistant Professor

Department of Anesthesiology

University of Maryland School of Medicine

22 S Greene Street, Baltimore, MD - 21201

PH - 4103281748

Manuscript Word Count: 2798

Keywords: echocardiographic, nonsystolic, diastolic dysfunction, transesophageal echocardiography, heart failure, coronary artery bypass, perioperative

\section{Key Points:}

Question: Is there a relationship between intra-operative nonsystolic echocardiographic parameters and outcome following coronary artery bypass (CAB) surgery?Findings: In this prospective observational study, severe dysfunction of non-systolic parameters, determined by intraoperative transesophageal echocardiography (TEE), was related in dose-response fashion to duration of postoperative hospital stay; those with abnormal parameters in non-systole(diastolic) despite normal systolic function, prior to coronary revascularization, had a 35-40\% longer hospital stay than those with normal diastolic function.Meaning: Abnormal left ventricular non-systolic parameters on intra-operative TEE is an independent determinant of hospital length of stay in patients with normal systolic function undergoing coronary artery bypass (CAB) surgery.

\section{INTRODUCTION}

Risk scoring systems for cardiac surgery patients first became popular with the Parsonnet score to calculate mortality risk [1]. Though subsequent evolution allows for calculation of postoperative morbidity [2] and 
reflects advances in our understanding of the patient's determinants for risk [3], these risk scoring systems remain imperfect $[4,5]$.

It is now recognized that cardiac performance during diastole influences morbidity [6] [7]. We have previously shown that, in patients undergoing coronary artery bypass (CAB) and/or Aortic Valvular surgery, diastolic function evaluated by preoperative Transthoracic Echocardiography (TTE) predicts hospital stay in patients with varying degrees of systolic function [8]. That study did not address the predictive capacity of transesophageal echocardiography (TEE), in which parameters of LV's nonsystolic function (e.g. LA max volume) are much less reliably [9-11] or less practically $[12,13]$ obtained.

This is a prospective cohort study involving subjects with normal systolic function undergoing CAB surgery to evaluate the association between intraoperative TEE metrics of LV performance during diastole and postoperative outcomes. The TEE algorithm depends on a limited number of metrics of performance during diastole and is validated to predict 5 year composite outcomes following CAB [14]. We hypothesized that the algorithm would be associated with duration of hospitalization after CAB.

\section{METHODS}

\section{Study Participants}

Patients who underwent CAB using cardiopulmonary bypass (CPB) at the Johns Hopkins Hospital (JHH) from January 2017 to July 2018 and underwent a TEE exam to assess diastolic function were eligible for inclusion. Individuals were excluded for any of the following: left ventricular ejection fraction $<50 \%$; preoperative electrical pacing, inotropic support, or mechanical ventricular support. Institutional approval by the Johns Hopkins Medical Institutional Review Board was received, and requirement for written informed consent was waived.

\section{Echocardiographic Assessment}

A protocol for evaluating performance during diastole by intraoperative TEE was introduced at JHH in January 2017 and intended for all patients undergoing CAB surgery. Examinations were performed after induction of anesthesia and prior to coronary artery revascularization, i.e. prior to CPB, though timing in relation to sternotomy or phase of respiratory cycle were not standardized. All TEEs were performed by physicians NBE-certified in advanced perioperative TEE or by cardiac anesthesia fellows under the direct supervision of a certified physician. Metrics of performance during diastole were interpreted by a single physician (JMD) blinded to each subject's preoperative comorbidities and postoperative course. The TEE evaluation of performance during diastole was modified from that of Swaminathan et al [14] and included: 1) spectral pulsed wave Doppler velocity of transmitral early (E) inflow; 2) spectral tissue Doppler imaging of diastolic myocardial velocity at the lateral mitral annulus (e'). Unless atrial fibrillation was present, the most representative waves were chosen. In the case of atrial fibrillation, values were averaged over 6-7 beats. Abnormal performance during diastole, hereafter referred to as diastolic dysfunction, was defined dichotomously as e' $<10 \mathrm{~cm} / \mathrm{s}$. If $\mathrm{LV}$ dysfunction during diastole existed unlike during systole when it was normal, severity was defined as: 1) Grade 1 if E/e' $<8.5 ; 2$ ) Grade 2 if E/e' 8.6-12.5; 3) Grade 3 if $\mathrm{E} / \mathrm{e}$ ' $>12.6$. These compromise $\mathrm{E} / \mathrm{e}$ ' cutoffs were chosen to accommodate noninteger values not specifically categorized in the reference manuscript [14]. Abnormal E was defined dichotomously as E $>50 \mathrm{~cm} / \mathrm{sec}$ [15].

\section{Baseline Covariates and Follow-up}

Baseline (prior to surgery) demographic characteristics, comorbidities, and laboratory data were extracted from the medical record. Medical care throughout the hospital course was at the direction of the clinical team, who recorded diagnoses (ICD-10 codes), laboratory tests, and medications as part of routine care. The clinical team was not blinded to results of the evaluation of performance during diastole. Follow-up included the intraoperative and postoperative care phases until the time of hospital discharge. Complications were determined from abstraction of data routinely recorded in the medical record. Intravenous fluids and red blood cells administered volumes were abstracted from the medical record from time of surgery through the $4^{\text {th }}$ postoperative day. 


\section{Outcome Measures}

The primary outcome, time to hospital discharge, was defined as the time from end of surgery to hospital discharge. Secondary outcomes were: interval until extubation (requiring greater than 6 hours following end of surgery), interval until free of supplemental oxygen (days from end of surgery to breathing room air), composite cardio-respiratory morbidity (i.e. postoperative diagnosis of heart failure, respiratory insufficiency, respiratory failure, or pulmonary edema), new atrial fibrillation, postoperative hypotension, hypervolemia, acute kidney injury (AKI; defined as maximal change in creatinine from baseline by KDIGO criteria) [16] , stroke, infection (surgical site infection [SSI], pneumonia, or sepsis), and readmission to the ICU.

\section{Statistical Analysis}

Continuous variables were summarized using mean \pm SD. Categorical variables were summarized using percentage. Variables were compared across subjects with normal and diastolic dysfunction using t-test, Kruskal Wallis, and chi-square test. Distinct clusters (heterogenous groups) within a patient sample were evaluated using latent class analysis (LCA) to classify subjects into multiple comorbidity groups based on comorbidities present at admission. LCA uses the joint distribution of observed responses across all individuals on a set of items (i.e., types of comorbidities) to characterize an underlying categorical latent variable that subdivides the given population into a smaller number of groups using modal class assignment. LCAs were conducted using the 12 baseline variables representing comorbidities based on cardiovascular diseases, chronic lung diseases, obesity and baseline ASA score. Since the number of clusters is unknown a priori, statistical comparisons of model fit, based primarily on the log likelihood value and Bayesian Information Criterion (BIC) were used to compare models with an increasing number of clusters. An LCA is particularly suitable because of its ability to specify unobserved (latent) subgroups of individuals [17]

Cox Proportional Hazard models, with the response variable being 'time to discharge' and the event variable being 'discharge', were used to evaluate the association between time to discharge and diastolic dysfunction with and without adjustment for confounding variables. Additional Cox models were used to determine the role of secondary outcomes in mediating the relationship between diastolic dysfunction and time to discharge. Survival analyses were performed using the $\mathrm{R}$ programming environment. All estimates and confidence intervals (CIs) were obtained using the "coxph" function.

\section{RESULTS}

TEE evaluation of function during diastole was inconsistently completed, but consistency increased over the course of the study, approaching $90 \%$ of eligible patients by the end of the study. Relative frequency of reasons for incomplete evaluation (eg. poor image alignment, excessive nonechocardiographic/clinical demands) could not be determined. Data were captured on 176 eligible subjects (mean age $65.2+/-9.2$ years, $73 \%$ male, $76 \%$ white). Baseline characteristics of study participants, with normal $(\mathrm{N}=105)$ vs abnormal $(\mathrm{N}=71)$ baseline performance during diastole, are shown in Table 1 . Those with abnormal echocardiographic parameters during nonsystole: 1) were older; 2) were more likely to be female, and/or have a history of congestive heart failure, valvular disease, and/or renal dysfunction; 3) trended toward a higher prevalence of prior myocardial infarction and history of chronic lung disease; 4) less likely to have hypertension; 5) trended towards a shorter duration of CPB. Overall, baseline characteristics suggested a greater burden of comorbidity at baseline for those with diastolic dysfunction, supported by significantly higher baseline ASA class, a composite comorbidity score well recognized to be associated with postoperative morbidity and mortality [18-20]. Based on the log-likelihood test and BIC, LCA classified subjects into two groups (high and low severity) based on baseline characteristics, and the burden of comorbid illness appeared to be greater in latent class 2 than 1 (Table 1 ). Subjects with diastolic dysfunction were more likely to be classified in latent class 2 than 1 (Table 1 ).

Median time to hospital discharge was significantly longer for subjects with abnormal vs normal performance during diastole (abnormal: 9.1/IQR 6.6-13.5 days; normal: 6.5/IQR 5.3-9.7 days) ( $\mathrm{P}<0.001$ by KruskalWallace test). Probability of hospital discharge on any given postoperative day (hereafter referred to as "daily probability of discharge") was significantly lower for those with diastolic dysfunction (Fig 1 ). There 
was a dose-response relationship between severity of diastolic dysfunction and daily probability of discharge - those having the most severely abnormal performance had the lowest daily probability of discharge (Fig $\mathbf{2}$ ) .

We examined the independent relationship of baseline diastolic dysfunction to daily probability of discharge in a series of unadjusted and adjusted Cox-Proportional Hazards models (Table 2 ) Daily probability of discharge was $46 \%$ lower (RR 0.54/95\% CI 0.40-0.75) for those with diastolic dysfunction compared to those with normal performance during diastole in unadjusted analysis. Adjusting for age, sex (Table 2, Model 1 ), duration of CPB (Table 2, Models 1, 2 ), severity of illness latent class (Table 2, Models 2, 3 ) history of heart failure and myocardial infarction (Table 2, Model 3 ), the daily probability of discharge remained significantly lower for those with diastolic dysfunction. A Sensitivity Analysis excluding 5 subjects with more than mild valvular disease (eg. MAC, Aortic disease, Mitral disease) did not affect these results (RR $0.63 / 95 \%$ CI 0.45-0.90). Our propensity analysis successfully matched 42 subjects with and without diastolic dysfunction, and showed that daily probability of discharge was $37 \%$ lower (RR 0.63/95\% CI 0.40-1.00) for those with diastolic dysfunction. Our LCA showed the daily probability of discharge: 1) was significantly lower for the group with both diastolic dysfunction and high severity of illness latent class (RR 0.39/95\% CI $0.26-0.58 ; \mathrm{P}<0.001) ; 2$ ) tended to be lower in the group with diastolic dysfunction and low severity of illness (RR 0.72/95\% CI 0.50-1.05; $\mathrm{P}=0.09$ ); 3) had no pattern of relation for the two strata with normal performance during diastole (Supplemental Fig 1 ). Finally, in additional models that included LV performance during diastole, $\mathrm{E}$ and the $\mathrm{E} / \mathrm{e}$ ' ratio as covariates in the regression model (Table 2, Model 3 ), the association between abnormal e' and discharge was unchanged (RR 0.61/ 95\% CI 0.42-0.88). Abnormal E was also independently associated with discharge (RR $0.61 / 95 \%$ CI0.39-0.94), but E/e' was not (RR $0.85 / 95 \%$ CI $0.48-1.52)$.

There were no significant differences in intravenous fluid administration $(6169 \pm 2958 \mathrm{ml}$ vs. $6229 \pm 3542 \mathrm{ml}$, $\mathrm{P}=0.99)$ or transfusion of packed red blood cells $(229 \pm 404 \mathrm{ml}$ vs. $187 \pm 377 \mathrm{ml} ; \mathrm{P}=0.48)$ between subjects with and without diastolic dysfunction. Secondary intermediate outcomes are shown in Table 3 . Subjects with diastolic dysfunction were more likely to be extubated $>6$ hours after surgery, to require supplemental oxygen longer, and to be diagnosed with the composite outcome of heart failure, respiratory insufficiency/failure, or pulmonary edema. We found no significant differences by performance during diastole in the incidences of postoperative atrial fibrillation, AKI, stroke, or infection. A higher proportion of subjects with diastolic dysfunction were readmitted to the ICU; however, this difference was not statistically significant (Table 3 ) .

The relationship of diastolic dysfunction and secondary intermediate outcomes to daily probability of discharge is shown in Table 4. Similar to baseline diastolic dysfunction, each of the secondary outcomes was associated with a lower daily probability of discharge in unadjusted analysis. In multivariable cox regression, secondary outcomes had modest effect on the relationship between baseline diastolic dysfunction and daily probability of discharge, which remained $37 \%$ less likely for those with diastolic dysfunction (Table 4 ).

\section{DISCUSSION}

This prospective, observational study, evaluated the association between non-systolic TEE metrics of left ventricular performance, assessed during intraoperative TEE before CPB, and interval to hospital discharge after $\mathrm{CAB}$. We found that abnormal left ventricular performance during diastole was: 1) associated with a significantly longer postoperative hospital stay, 2) associated with a greater baseline comorbidity burden. Still, multivariate analysis showed their $35-40 \%$ lower daily probability of discharge (compared to subjects with normal performance during diastole) was independent of baseline comorbidities, including heart failure and myocardial infarction.; 3) dose-dependently related to daily probability of discharge; and, 4) associated with numerous complications after surgery, particularly heart failure and respiratory insufficiency/failure. These complications did not fully account for the relationship between diastolic dysfunction and daily probability of discharge.

Compared to patients with normal performance during diastole, we observed a $31 \%$ longer mean hospital 
stay in patients with diastolic dysfunction. This does not appear to be an artifact driven by outliers, given the nature of the nonparametric Kruskal Wallis evaluation performed. The unadjusted ratio for daily probability of discharge in a patient with diastolic dysfunction is 0.54 . This suggests a $46 \%$ lower daily probability of discharge if a patient has any degree of diastolic dysfunction (vs normal performance during diastole). We have previously demonstrated that, independent of systolic function, preoperative TTE-measured diastolic dysfunction is associated with prolonged hospitalization as part of a combined endpoint in patients undergoing CAB, Aortic Valve replacement, or combined CAB and Aortic Valve replacement [8]. Severe diastolic dysfunction, determined by transmitral (E/A ratio, E wave deceleration time) and pulmonary venous flow patterns obtained by pre-operative TTE, has been shown to predict the occurrence of low output states within 30 days of $\mathrm{CAB}$ surgery [21]. The present study demonstrates that, in patients undergoing $\mathrm{CAB}$ surgery alone who have preserved systolic function, the presence of any degree of diastolic dysfunction on baseline intraoperative TEE is associated with prolonged post-CAB hospitalization as an isolated endpoint.

The graded association between abnormal non-systolic TEE metrics and prolonged post-CAB hospital stay that we observed in this study mimics the graded association between diastolic dysfunction and event-free five year survival reported following CAB surgery [14]. Both studies had comparable patient comorbidity patterns and utilized similar echocardiographic analysis of performance during diastole. In our study, the prolongation in hospital stay persisted even after adjusting for all baseline co-morbidities (including heart failure). This suggests that classic co-morbidity focused pharmacotherapeutic interventions will fail to modify hospital stay following $\mathrm{CAB}$ in patients with normal systolic function but diastolic dysfunction in a manner similar to their failure to modify long-term morbidity in patients with preserved systolic function, diastolic dysfunction and a history of heart failure. [22-26] .

The relevance of the E/e' ratio as an indicator of left sided filling pressures [15, 27-29] is one of great debate and beyond the scope of our study. We note that, regardless of the E/e' ratio, the presence of either $\mathrm{E}>50$ $\mathrm{cm} / \mathrm{sec}$ or e' $<10 \mathrm{~cm} / \mathrm{sec}$ is associated with a lower daily probability of discharge following CAB surgery. We found no interdependence of $\mathrm{E}>50 \mathrm{~cm} / \mathrm{sec}$ and $\mathrm{e}^{\prime}<10 \mathrm{~cm} / \mathrm{sec}$ with regard to daily probability of discharge, suggesting that these individual metrics reflect distinct phenomenon. An E $>50 \mathrm{~cm} / \mathrm{sec}$ indicates that left atrial pressure is elevated in relation to LV diastolic pressure, regardless of ease of LV relaxation. Similarly, a slow e' suggests that early LV relaxation is impaired, regardless of LA:LV pressure relationship [15]. The existence of either one of these conditions (E $>50 \mathrm{~cm} / \mathrm{sec}$ or e' $<10 \mathrm{~cm} / \mathrm{sec}$ ), regardless of the value of the other parameter, is associated with a lower daily probability of discharge in our study. The E/e' ratio does not improve upon these associations.

There are several limitations to our prospective cohort study. First, protocol adherence was inconsistent over the course of the study. Limited feedback with respect to obstacles to adherence prohibits a true understanding as to the breadth of the protocol's utility. The small study population restricts subgroup size, preventing utilization of a more robust statistical analysis. Thus, while our LCA and the propensity score analyses indicate that co-morbidities do not account for the entire effect of baseline diastolic dysfunction on hospital stay, a large number of our propensity score subjects did not have good matches. The study population size may also obscure the identification of an interaction between diastolic dysfunction and a perioperative morbidity. Finally, the true value of E/e' remains unclear. Although originally defined as a noninvasive approach to evaluate left heart filling pressures [30], this concept has been challenged [29]. In our study, E/e' does not add to the predictive capacity of $\mathrm{E}>50 \mathrm{~cm} / \mathrm{sec}$ or e' $<10 \mathrm{~cm} / \mathrm{sec}$ with regard to presence or absence of lower daily probability of hospital discharge following $\mathrm{CAB}$, but does show a graded association with the degree to which daily probability of hospital discharge is lowered. It has previously been shown to correlate with long term risk of major cardiac adverse effects [7]. In that study, as in ours, the study population was patients undergoing CAB surgery with baseline LVEF $>50 \%$.

\section{CONCLUSION}

In conclusion, we found that, compared to normal intraoperative TEE metrics during diastole prior to $\mathrm{CPB}$, abnormal non-systolic echocardiographic parameters are associated with a $31 \%$ longer hospital stay following $\mathrm{CAB}$ surgery in patients with normal baseline left ventricular systolic function. In this case, diastolic 
dysfunction is indicated by the presence of either E $>50 \mathrm{~cm} / \mathrm{sec}$ or e' $<10 \mathrm{~cm} / \mathrm{sec}$. The daily probability of discharge is inversely related to the severity of performance abnormality during diastole in a dose-dependent manner. The prolongation in hospital stay could be accounted for neither by baseline comorbidities nor by post-op complications. Larger studies are needed to confirm the consistency of these results and to elucidate a modifiable cause of the prolonged hospital stay.

\title{
Table Legends
}

Table1: Characteristics of study sample for subjects with abnormal and normal pre-surgical performance during diastole.

Table 2. Adjusted and un-adjusted rate ratios for discharge from the hospital using cox proportional hazard model

Table 3. Secondary outcomes for subjects with abnormal and normal pre-surgical performance during diastole.

Table 4 Relation of baseline abnormal nonsystolic function and secondary outcomes to time to hospital discharge

\section{Figure Legends}

Figure 1 -Cox Proportional Hazard Model for Daily Probability of Discharge vs Time (days) following surgery. A. Subjects with abnormal (blue) vs. normal (red) left ventricular performance during diastole. B. Dose-response relationship between severity of performance abnormality during diastole and daily probability of discharge; mild dysfunction (green), moderate dysfunction (blue), severe dysfunction (purple), normal (red). Survival analyses performed using R programming environment. Estimates and CIs obtained using the "coxph"function.

Figure 2 - Daily probability of discharge over time in days by interaction between latent class and performance abnormality during diastole (before surgery)/normal status using Cox Proportional Hazard model

Figure 3: Probability of discharge over time in days by interaction between pre-surgical performance during diastole (normal/abnormal) and latent class (low/high severity) using Cox Proportional Hazard model

\author{
Glossary of terms - \\ BIC - Bayesian Information Criterion \\ CAB - Coronary artery bypass \\ CPB - Cardiopulmonary bypass \\ E - Transmitral pulse wave doppler velocity during diastole \\ E' - Tissue doppler velocity at lateral mitral annulus during diastole \\ $\mathrm{EF}-$ Ejection fraction \\ HF - Heart failure \\ LV - Left ventricle \\ LCA - Latent Class Analysis \\ RV - Right ventricle \\ TEE - Transesophageal echocardiography \\ TTE - Transthoracic echocardiography
}

\section{Declaration:}




\section{Ethics approval and consent to participate -}

Institutional approval by the Johns Hopkins Medical Institution Review Board was received, and requirement for written informed consent was waived.

\section{Consent for publication -}

Not applicable

\section{Availability of data and materials}

All data were de-identified and no personal data was stored. Data was stored in encrypted institutional computers and devices for analysis purpose. The datasets during and/or analyzed during the current study available from the corresponding author on reasonable request.

- Competing interests - None of the authors have any competing interests

- Funding: Not applicable; all costs and charges were borne by researchers professional developmental fund

\section{- Authors' contributions -}

SM - Corresponding author; data collection, writing the manuscript NF - Data analysis, manuscript writing WG - Manuscript writing, data analysis SS- Data analysis SH - Data analysis KNH - Data collection, revising the critical content of the manuscript TSM - Manuscript writing, designing the study LG - Study design MB - Data collection BB - Data collection BC - Data collection SC - Data collection DM - Data collection SOI - Data collection, revising the critical content of the manuscript NR - Data collection LL Data Analysis, study design JS - Manuscript writing, data collection RS - Manuscript writing, data collection NH - Data Collection AK - Data collection JMD - Principal investigator, Study design, Manuscript writing, Data Analysis

Acknowledgements - Not applicable

\section{References}

1. Parsonnet, V., D. Dean, and A.D. Bernstein, A method of uniform stratification of risk for evaluating the results of surgery in acquired adult heart disease.Circulation, 1989. 79 (6 Pt 2): p. I3-12.

2. Granton, J. and D. Cheng, Risk stratification models for cardiac surgery. Semin Cardiothorac Vasc Anesth, 2008. 12 (3): p. 167-74.

3. Rosenhek, R., et al., ESC Working Group on Valvular Heart Disease Position Paper: assessing the risk of interventions in patients with valvular heart disease. Eur Heart J, 2012. 33 (7): p. 822-8, 828a, 828b.

4. Ad, N., et al., Comparison of EuroSCORE II, Original EuroSCORE, and The Society of Thoracic Surgeons Risk Score in Cardiac Surgery Patients. Ann Thorac Surg, 2016.102 (2): p. 573-9.

5. Prins, C., et al., Cardiac surgery risk-stratification models. Cardiovasc J Afr, 2012.23 (3): p. 160-4.

6. AlJaroudi, W.A., et al.,Prognostic value of diastolic dysfunction: state of the art review. Cardiol Rev, 2014. 22 (2): p. 79-90.

7. Swaminathan, M., et al., Utility of a simple algorithm to grade diastolic dysfunction and predict outcome after coronary artery bypass graft surgery. Ann Thorac Surg, 2011. 91 (6): p. 1844-50.

8. Metkus, T.S., et al.,Diastolic dysfunction is common and predicts outcome after cardiac surgery. J Cardiothorac Surg, 2018. 13 (1): p. 67.

9. Singh, H., et al., Comparison of left atrial dimensions by transesophageal and transthoracic echocardiography. Echocardiography, 2005. 22 (10): p. 789-96.

10. Eshoo, S., D.L. Ross, and L. Thomas, Evaluation of left atrial size on transoesophageal echocardiography: what is the best measure? Heart Lung Circ, 2008.17 (2): p. 100-6. 
11. Ancona, R., et al., Left atrium by echocardiography in clinical practice: from conventional methods to new echocardiographic techniques. ScientificWorldJournal, 2014. 2014 : p. 451042.

12. Cameli, M., et al., Novel echocardiographic techniques to assess left atrial size, anatomy and function. Cardiovasc Ultrasound, 2012. 10 : p. 4.

13. Mor-Avi, V., et al.,Real-time 3D echocardiographic quantification of left atrial volume: multicenter study for validation with CMR. JACC Cardiovasc Imaging, 2012. 5 (8): p. 769-77.

14. Swaminathan, M., et al., Utility of a simple algorithm to grade diastolic dysfunction and predict outcome after coronary artery bypass graft surgery. Ann Thorac Surg, 2011. 91 (6): p. 1844-50.

15. Nagueh, S.F., et al.,Recommendations for the Evaluation of Left Ventricular Diastolic Function by Echocardiography: An Update from the American Society of Echocardiography and the European Association of Cardiovascular Imaging. J Am Soc Echocardiogr, 2016. 29 (4): p. 277-314.

16. Roy, A.K., et al., A Comparison of Traditional and Novel Definitions (RIFLE, AKIN, and KDIGO) of Acute Kidney Injury for the Prediction of Outcomes in Acute Decompensated Heart Failure. Cardiorenal Med, 2013. 3 (1): p. 26-37.

17. Muthen, B. and L.K. Muthen,Integrating person-centered and variable-centered analyses: growth mixture modeling with latent trajectory classes. Alcohol Clin Exp Res, 2000. 24 (6): p. 882-91.

18. Wolters, U., et al., ASA classification and perioperative variables as predictors of postoperative outcome. Br J Anaesth, 1996. 77 (2): p. 217-22.

19. Hackett, N.J., et al., ASA class is a reliable independent predictor of medical complications and mortality following surgery. Int J Surg, 2015. 18 : p. 184-90.

20. Somani, S., et al., ASA Classification as a Risk Stratification Tool in Adult Spinal Deformity Surgery: A Study of 5805 Patients. Global Spine J, 2017. 7 (8): p. 719-726.

21. Merello, L., et al., Risk scores do not predict high mortality after coronary artery bypass surgery in the presence of diastolic dysfunction. Ann Thorac Surg, 2008. 85 (4): p. 1247-55.

22. Yusuf, S., et al., Effects of candesartan in patients with chronic heart failure and preserved left-ventricular ejection fraction: the CHARM-Preserved Trial. Lancet, 2003. 362 (9386): p. 777-81.

23. Cleland, J.G., et al., The perindopril in elderly people with chronic heart failure (PEP-CHF) study. Eur Heart J, 2006. 27 (19): p. 2338-45.

24. Massie, B.M., et al.,Irbesartan in patients with heart failure and preserved ejection fraction. N Engl J Med, 2008. 359 (23): p. 2456-67.

25. Pitt, B., et al.,Spironolactone for heart failure with preserved ejection fraction. N Engl J Med, 2014. 370 (15): p. 1383-92.

26. Redfield, M.M., et al.,Effect of phosphodiesterase-5 inhibition on exercise capacity and clinical status in heart failure with preserved ejection fraction: a randomized clinical trial. JAMA, 2013. 309 (12): p. 1268-77.

27. Okada, D.R., et al.,Clinical echocardiographic indices of left ventricular diastolic function correlate poorly with pulmonary capillary wedge pressure at 1 year following heart transplantation. Int J Cardiovasc Imaging, 2015.31 (4): p. 783-94.

28. Ommen, S.R., et al.,Clinical utility of Doppler echocardiography and tissue Doppler imaging in the estimation of left ventricular filling pressures: A comparative simultaneous Doppler-catheterization study. Circulation, 2000. 102 (15): p. 1788-94.

29. Sharifov, O.F., et al.,Diagnostic Accuracy of Tissue Doppler Index E/e' for Evaluating Left Ventricular Filling Pressure and Diastolic Dysfunction/Heart Failure With Preserved Ejection Fraction: A Systematic 
Review and Meta-Analysis. J Am Heart Assoc, 2016. 5 (1).

30. Nagueh, S.F., et al.,Doppler tissue imaging: a noninvasive technique for evaluation of left ventricular relaxation and estimation of filling pressures. J Am Coll Cardiol, 1997. 30 (6): p. 1527-33.

Hosted file

Figure_combined.docx available at https://authorea.com/users/409644/articles/539327echocardiographic-parameters-of-left-ventricular-non-systolic-function-predict-lengthof-stay-following-coronary-artery-bypass-graft-a-prospective-observational-study 